\title{
Successful Long-Term Airway Stabilization With a Modified Pacifier in a Syndromic Infant
}

\author{
Mehmet N Cizmeci MD, Mehmet K Kanburoglu MD, \\ Selma Ziraman RNP, and Mustafa M Tatli MD
}

\begin{abstract}
Airway management is one of the key aspects of neonatal anesthesia, and an oropharyngeal airway is used in daily intensive care practice to relieve upper-airway obstruction. Cleft palate is among the various congenital malformations that can cause difficult airway management. We describe a syndromic infant with alobar holoprosencephaly, whose long-term airway patency was maintained with a modified infant pacifier. We would like to share our experience and contribute to the literature with the introduction of a readily available and easily-inserted apparatus. Key words: airway stabilization; neonate; oropharyngeal airway; syndromic infant. [Respir Care 2014;59(2):e13-e14. (C) 2014 Daedalus Enterprises]
\end{abstract}

\section{Introduction}

Airway management is one of the key aspects of neonatal anesthesia, and an oropharyngeal airway is used in daily intensive care practice to relieve upper-airway obstruction. An oropharyngeal airway is a medical apparatus used to maintain a patent airway by preventing the tongue from obstructing the aperture of the upper airway. ${ }^{1,2}$

Cleft palate is among the conditions that can cause difficult airway management, due to inability to optimally position the head, and to maldevelopment, which makes it difficult to obtain a good view of the upper airway structures. ${ }^{3,4}$ We describe a syndromic infant with alobar holoprosencephaly, whose long-term airway patency was maintained with a modified infant pacifier.

For this type of case report no ethics committee approval is required in Turkey, but informed consent was obtained from the parents.

\footnotetext{
The authors are affiliated with the Division of Neonatology, Department of Pediatrics, Fatih University Medical School, Ankara, Turkey.

The authors have disclosed no conflicts of interest.

Correspondence: Mehmet Nevzat Cizmeci MD, Division of Neonatology, Department of Pediatrics, Fatih University Medical School, 06510, Emek, Ankara, Turkey. E-mail: nevzatcizmeci@gmail.com.
}

DOI: $10.4187 /$ respcare. 02530

\section{Case Report}

Our patient was a male infant born at 40 weeks gestation to a 24 -year-old, gravida 1 , para 1 , healthy mother, via cesarean section. During the first trimester of pregnancy the infant was detected to have holoprosencephaly and substantial hydrocephalus. There was no consanguinity, and the pregnancy history was unremarkable. Upon delivery the infant was admitted to the neonatal ICU for life support.

There was substantial microcephaly and facial dysmorphia, including a blind-ended single nostril, ocular hypotelorism, and complete cleft palate, all of which were consistent with cebocephaly. The rest of the physical examination was unremarkable. Magnetic resonance imaging of the cranium was obtained to guide the modality of airway stabilization, which revealed an osseous septum in the posterior nasopharynx and intracranial abnormalities consistent with alobar holoprosencephaly. Echocardiography and abdominal ultrasonography were unremarkable, but electroencephalography revealed diffuse repetitive electrographic seizures, which responded well to phenobarbital.

We consulted the otolaryngology clinic for selection of an airway stabilization modality and possible surgery. The otolaryngology council deemed the patient not a candidate for surgery, owing to the risks and the patient's very short life expectancy.

Since the infant was an obligate mouth-breather and desaturated rapidly in the absence of an airway interven- 


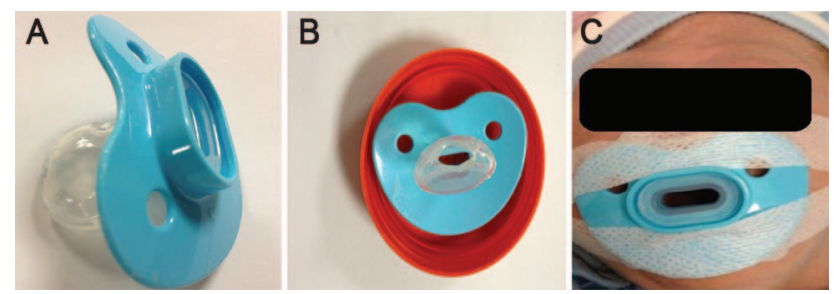

Fig. 1. A: Oropharyngeal airway made from an infant pacifier. B: The bulbous portion of the pacifier was trimmed to create an airway (in this image the device was placed on a red surface to highlight the airway conduit). C: Infant being adapted to the modified pacifier.

tion, an oropharyngeal airway was used to obtain airway patency. We then replaced the first oropharyngeal airway with a polyethylene oropharyngeal airway made from an infant pacifier (Fig. 1). The device has a hole in the middle of the distal tip of the bulbous region. The pacifier oropharyngeal airway we selected was one size larger than is recommended for our patient's age, because we needed the airway to extend further into the mouth than would the recommended pacifier size. The device provides airway patency, does not collapse under the force of the infant's bite, is easily tolerated by an unsedated infant, and appears to have a soothing effect. Our patient's oxygen saturation stabilized at $>95 \%$ on room air, without any further intervention. Since the infant was an obligate mouth breather, a percutaneous gastrostomy tube was placed for nutrition, and he was discharged on the 21 st postnatal day, to be followed up in a multidisciplinary out-patient clinic.

\section{Discussion}

Airway devices play an important role in the acute management of patients, and they are especially important in managing the difficult airway in the operating room and in the pre-hospital setting. ${ }^{5}$ Novel airway devices are becoming widely used in routine practice. ${ }^{4}$ While we need to research these novel technologies, we should also dissem- inate simple and practical approaches that improve patients' quality of life, including infants with chronic and lethal conditions. An oropharyngeal airway is generally preferred for short-term airway management. Our aim with the modified infant pacifier was to prepare an infant with a major neurologic disability for discharge from the hospital, since the infant had an osseous septum in the posterior nasopharynx and could not tolerate the removal of an oropharyngeal airway. The pacifier device was well tolerated by the infant and maintained long-term upperairway patency.

After discharge the patient's parents were able to maintain the use of the pacifier device at home, and the infant was seen regularly in the out-patient clinic for follow-up visits. Repeated attempts to remove the pacifier device all resulted in acute respiratory distress. The infant was alive at age 5 months, but suffering from frequent upper-respiratory-tract infections, which might be attributable to the artificial patency of the airways.

We believe that some unconventional approaches and devices can improve the quality of life of patients with severe conditions and short life expectancy, and the pacifier oropharyngeal airway helped our patient and is readily available and easily inserted.

\section{REFERENCES}

1. Restrepo C, Santamaria A, Pelaez S, Tapias A. Oropharyngeal airway dimensions after treatment with functional appliances in class II retrognathic children. J Oral Rehabil 2011;38(8):588-594.

2. Kim SH, Kim DH, Kang H, Suk EH, Park PH. Estimation of teethto-vallecula distance for prediction of optimal oropharyngeal airway length in young children. Br J Anaesth 2011;107(5):769-773.

3. Mahmoud AA, Fouad AZ, Mansour MA, Kamal AM. A novel intubation technique in bilateral cleft palate pediatric patients: hard gum shield-aided intubation. Paediatr Anaesth 2013;23(4):349-354.

4. Ramesh S, Jayanthi R, Archana S. Paediatric airway management: what is new? Indian J Anaesth 2012;56(5):448-453.

5. Timmermann A. Supraglottic airways in difficult airway management: successes, failures, use and misuse. Anaesthesia 2011;66(Suppl 2):45-56. 